\title{
Characterization of Coupled Ground State and Excited State Equilibria by Fluorescence Spectral Deconvolution
}

\author{
Wouter Caarls • M. Soledad Celej • \\ Alexander P. Demchenko • Thomas M. Jovin
}

Received: 14 May 2009 / Accepted: 25 August 2009/Published online: 23 September 2009

(C) The Author(s) 2009. This article is published with open access at Springerlink.com

\begin{abstract}
Fluorescence probes with multiparametric response based on the relative variation in the intensities of several emission bands are of great general utility. An accurate interpretation of the system requires the determination of the number, positions and intensities of the spectral components. We have developed a new algorithm for spectral deconvolution that is applicable to fluorescence probes exhibiting a twostate ground-state equilibrium and a two-state excited-state reaction. Three distinct fluorescence emission bands are resolved, with a distribution of intensities that is excitationwavelength-dependent. The deconvolution of the spectrum into individual components is based on their representation as asymmetric Siano-Metzler log-normal functions. The application of the algorithm to the solvation response of a 3-hydroxychromone $(3 \mathrm{HC})$ derivative that exhibits an H-bonding-dependent excited-state intramolecular proton transfer (ESIPT) reaction allowed the separation of the spectral signatures characteristic of polarity and hydrogen bonding. This example demonstrates the ability of the method
\end{abstract}

\section{W. Caarls $\cdot$ T. M. Jovin $(\bowtie)$}

Laboratory of Cellular Dynamics,

Max Planck Institute for Biophysical Chemistry,

Am Fassberg 11,

D37077 Göttingen, Germany

e-mail: tjovin@gwdg.de

M. Soledad Celej

Departamento de Química Biológica-CIQUIBIC,

Facultad de Ciencias Químicas,

Universidad Nacional de Córdoba,

Haya de la Torre S/N, X5000HUA, Ciudad Universitaria,

Córdoba, Argentina

A. P. Demchenko

A. V. Palladin Institute of Biochemistry,

9 Leontovicha Str.,

01030 Kiev, Ukraine to characterize two potentially uncorrelated parameters characterizing dye environment and interactions.

Keywords Fluorescence deconvolution.

Excited state intramolecular proton transfer.

Hydrogen bonding $\cdot$ Polarity $\cdot$ Multiparametric probe

\section{Introduction}

Ground-state and excited-state interactions and transformations can generate multiple bands in the absorption and fluorescence spectra of organic fluorophores. These effects depend on the properties of the surrounding medium and may be used as extremely sensitive means for characterizing these properties in the systematic application of fluorescence sensors and probes [1]. One can detect and characterize numerous molecular events based on probe sorption-desorption to surfaces, inclusion into micelles and biomembranes, and attachment to proteins exhibiting conformational changes, self-assembly or aggregation. Increasing the number of spectrally resolved components and tailoring their sensitivity to different types of interactions dramatically increases the information content of fluorescence responses. However, such a multiparametric probing of intermolecular interactions [2] requires deconvolution of complex fluorescence responses into individual components and proper analysis of systematic changes of these components.

Of particular interest in this respect are the classes of organic dyes displaying two emissive ground-state forms, one of which can undergo an excited-state transformation with the generation of an additional fluorescence band, leading to three emission bands (Fig. 1). Generation of the response signal can be obtained in several ways: from 
the shift in ground-state equilibrium between reactive and nonreactive forms, from the shift in equilibrium between reactants and products in the excited-state reaction, and from the positions (also, polarizations and lifetimes) of all three fluorescence bands. Due to their differential sensitivities to different types of intermolecular interactions, these spectroscopic changes provide multiparametric information. In such probes the ground-state forms can be of different origin. They can be two conformers, or two species existing in protic equilibrium or directly or indirectly engaged in specific interactions involving charge-transfer complexes and H-bonds. The excitedstate transformations generating emissive species can be intramolecular and intermolecular proton transfer, and the transfer of electronic charge.

The differences in the positions of the corresponding fluorescence bands relative to their bandwidths are often rather small, resulting in substantial overlap and thereby creating difficulties in the estimation of peak positions and intensities. For a detailed characterization of spectral changes, deconvolution of complex spectra into individual components is required. However, such a transformation of a complex signal into individual components involves a certain degree of ambiguity because the result is unstable against slight and inevitable variations in the input data (systematic and/or random error). Thus, the solution of such so-called "reverse problems of spectroscopy" is mathematically ill-defined. No general methods exist, such that a reduction of ambiguity in the results requires additional constrains, i.e. 'regularizations' (functions, algorithms, or logical premises) [3].

Of central importance for appropriate deconvolution is the choice of the function describing the shape(s) of individual bands. The fluorescence spectra of organic dyes are usually broad and asymmetric [4]. The origin of this asymmetry is the involvement of electronic as well as electronic-vibrational (vibronic) transitions. The asymmetry in absorption spectra arises from the interplay of Franck-Condon factors: usually the $0-0$ band or closely located vibronic band is the most intensive, and the series of less intensive vibronic bands extend to higher energies. Due to inhomogeneous broadening [5], this vibronic structure is often hidden and one observes only a single asymmetric band with a steep red (long-wavelength) slope and a gentle short-wavelength slope. Ideally, the fluorescence spectra should be, and often are, mirror images of the absorption spectra, with sharp blue and gradual red slopes. In addition, they can be distorted by different relaxation processes in the excited state giving rise to Stokes shifts and additional spectral broadening. However, in the absence of fundamental physical laws requiring absorption or fluorescence bands to be symmetric, their approximation by symmetric Gaussian functions (as it is successfully done in IR and Raman spectroscopy) is not justified. Such procedures do not yield good fits to experimental bands [6], and moreover, may even lead to false additional bands on gradual slopes [7]. Therefore, in addition to three common parameters describing each spectral band (position and intensity of the maximum, width), one needs to account for variable band asymmetry. In these cases the Siano-Metzler log-normal distribution function [8] is generally used [9-12].

The present paper addresses the problem of deconvolution of spectra in which a two-state equilibrium in the ground state is coupled to an excited-state reaction producing two species with distinctive emission bands. The developed algorithm is based on the $2 \mathrm{D}$ analysis of the excitation-emission manifold. It uses as an input fluorescence spectra obtained by wavelength-selective excitation and an approximation of individual bands by log-normal functions. We show that curve-fitting based on deconvolution into log-normal components provides a reliable physical modeling of the probe response to intermolecular interactions. Two channels of information are derived, based on the independent detection of ground-state and excited-state equilibria. In the case of a dye exhibiting a reversible excited-state intramolecular proton transfer (ESIPT) reaction, the procedure allows the estimation of the effects of polarity and hydrogen bonding based on the fluorescence response.

\section{Deconvolution algorithm}

Approximation of spectra with log-normal distributions

The Siano-Metzler asymmetric log-normal function (alogn) is given by Eq. 1 and illustrated in Fig. 2. It has four parameters: the peak position in wavenumber $\nu_{0}$, full width at half maximum $H$, asymmetry $\rho$, and height $I_{0}$.

$\operatorname{alogn}\left(v_{0}, H, \rho, I_{0}, v\right)=I_{0} e^{-\left(\frac{\ln 2}{\ln \rho^{2}}\right)\left(\ln \left[\left(\frac{v-v_{0}}{H}\right)\left(\frac{\rho^{2}-1}{\rho}+1\right)\right]\right)^{2}}$

Each spectral band is approximated by one such function, and the total fluorescence spectrum is given by the sum of individual functions. To simplify the interpretation of the parameters, our plots and tables report band positions in wavelength instead of wavenumber, with $\lambda_{0}$ $(\mathrm{nm})=10^{7} / \nu_{0}\left(\mathrm{~cm}^{-1}\right)$.

Introduction of constraints

In order to reduce ambiguity and thus permit the accurate determination of the position, height, and width for each band contributing to the multi-component fluorescence 
spectrum, we apply a series of constraints to the data and the deconvolution procedure:

1. The component bands in excitation as well as in emission should conform to Siano-Metzler asymmetric log-normal functions.

2. The component positions and shapes in the excitation spectra should not depend on emission wavelength, and the component positions and shapes in emission spectra should not depend on excitation wavelength. (Deviations from this rule known as the red-edge effects [13] will not be considered here.)

3. The experimental excitation and emission spectra should be linear combinations of the overlapping band shapes of the components.

4. The number of components should be limited to two in the excitation and three in the fluorescence emission spectra. This condition derives from the known mechanisms of the class of ground-state and excitedstate reactions under consideration.

5. The ratio of the peak emission intensities of components 1 and 2 that derive from a single ground-state species (see Fig. 1) should not depend on the excitation wavelength.

6. The asymmetry, FWHM, and position of the three bands should be constrained to a physically plausible range.
These ranges can be derived from single-band fits to the spectrum of the dye in solvents in which it exhibits only single-band fluorescence. Since this is difficult to achieve with the present class of compounds, two bands with reasonably good separation can also be used.

\section{Global 2D deconvolution algorithm}

As we are modeling both the excitation spectra $I\left(\lambda_{\mathrm{ex}}\right)$ and emission spectra $I\left(\lambda_{\mathrm{em}}\right)$, we incorporate them into a twodimensional manifold $I\left(\lambda_{\mathrm{ex}}, \lambda_{\mathrm{em}}\right)$ describing the excitationwavelength dependence of the emission spectra due to multi-band excitation. A set of fluorescence spectra is taken at different excitation wavelengths and a two-dimensional fitting procedure across all these excitation wavelengths allows the proper deconvolution of the data. Constraints are applied, in particular the independence on $\lambda_{\mathrm{ex}}$ of the intensity ratio of components 1 and 2 of the fluorescence spectra.

The model function is given in Eq. 2. Each band $b$ is modeled with seven parameters: three for the shape of its emission spectrum $\left(v_{0, \mathrm{em}}^{b}, H_{\mathrm{em}}^{b}, \rho_{\mathrm{em}}^{b}\right)$ and four for the shape and height of its excitation spectrum $\left(v_{0, \mathrm{ex}}^{b}, H_{\mathrm{ex}}^{b}, \rho_{\mathrm{ex}}^{b}, I_{0}^{b}\right)$. $I\left(\lambda_{\text {ex }}, \lambda_{\text {em }}\right)$ is a linear combination of these bands, fitted in the wavenumber domain.

$I\left(\lambda_{\mathrm{ex}}, \lambda_{\mathrm{em}}\right)=\sum_{b \in \text { bands }} \operatorname{alogn}\left(v_{0, \mathrm{em}}^{b}, H_{\mathrm{em}}^{b}, \rho_{\mathrm{em}}^{b}, \operatorname{alogn}\left(v_{0, \mathrm{ex}}^{b}, H_{\mathrm{ex}}^{b}, \rho_{\mathrm{ex}}^{b}, I_{0}^{b}, \frac{10^{7}}{\lambda_{\mathrm{ex}}}\right), \frac{10^{7}}{\lambda_{\mathrm{em}}}\right)$

For three bands, this results in 21 parameters. However, due to the constraints given in the previous section, the shape of the excitation spectrum for the 1 st and 2 nd components is identical, with only $I_{0}^{b}$ being different. The final number of parameters is thus 18 . If the band with the shifted excitation spectrum is absent (characterized by a fitted shift of less than $2 \mathrm{~nm}$ ), we perform a 2-band fit instead, with 11 parameters. This avoids nonphysical results due to fitting a model with more parameters than is actually supported by the data. Figure 3 illustrates the twodimensional model function for three bands. The predominant (green squares) band excitation spectrum (projected on the right plane) is red-shifted with respect to the others, while its emission spectrum (projected on the back plane) is in between. The function is defined for any combination of $\lambda_{\text {ex }}$ and $\lambda_{\text {em. }}$.

The parameters were initialized randomly in a suitable range, and were allowed to vary according to the following
Fig. 1 General scheme of ground-state and excited-state transformations and emissions in the case of a reversible excited-state reaction and two ground state species

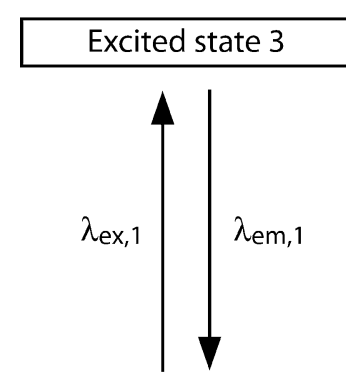

Ground state 3

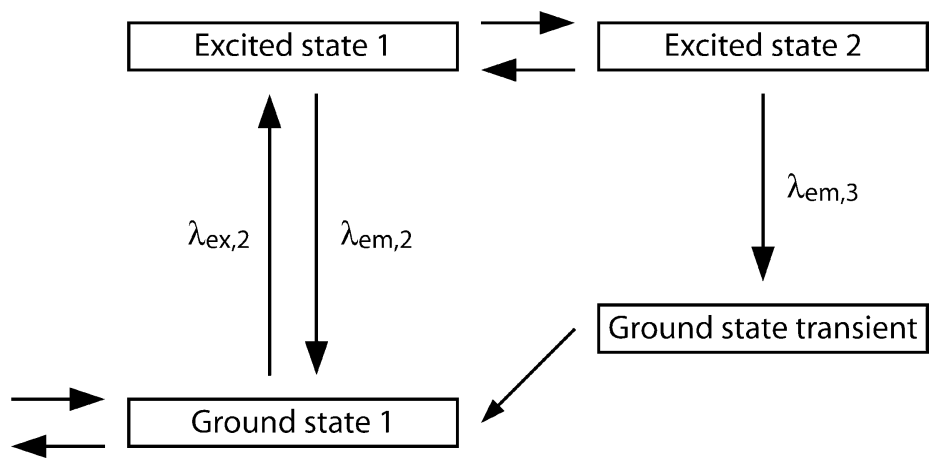




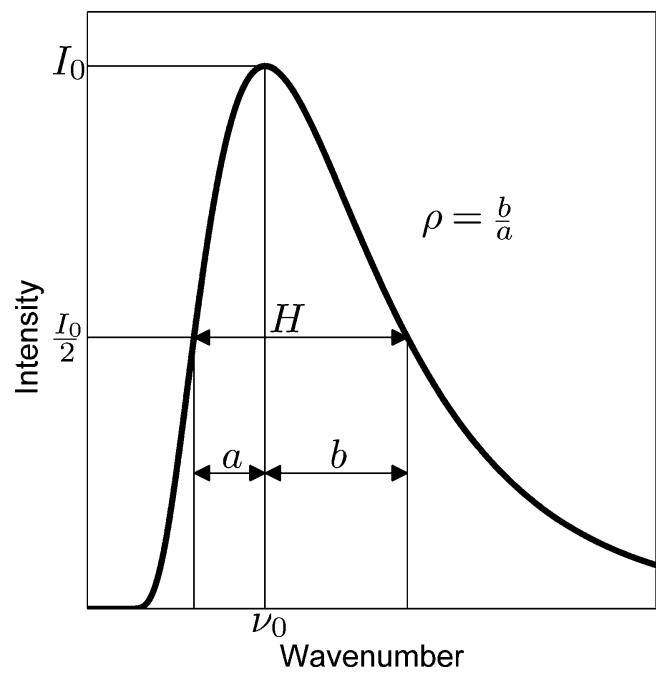

Fig. 2 Parameters of the log-normal function: peak position $\nu_{0}$, full width at half maximum $H$, asymmetry $\rho$ and height $I_{0}$

constraints: $0.5 \leq \rho_{\mathrm{em}} \leq 1,1 \leq \rho_{\mathrm{ex}} \leq 1.5$, and $500 \leq H \leq 5,500$. The fitting was performed using the iterative non-linear least squares Levenberg-Marquardt technique provided by the MATLAB programming language.

Comparison of areas under spectral components

From the deconvolved spectra we can characterize the ground state and excited state equilibria. The ratio of the areas under the bands participating in the excited-state equilibrium is a direct measure of the solvent parameter affecting the given reaction. The situation for the groundstate equilibrium is somewhat more complicated, because one of the states produces two emission bands that need to be merged. Simple addition of the areas is only possible if their quantum yields are equal. If that is the case, or if the relative quantum yields are known, the solvent parameter characterizing the ground-state equilibrium can be measured as the ratio between the area under the nonreactive excited state band and the weighted sum of the areas under the two reactive excited-state bands.

Since the individual components in the spectra are of different widths, the areas under the resolved component spectra are considered to provide better estimates of the number of quanta emitted from the corresponding states than the intensities at single wavelengths, which were used previously $[2,14]$. In addition, area calculations compensate for correlated errors in the dual parametric intensitywidth estimations and are thus more resilient to fitting errors than peak intensities. That is, we consider the area under the individual emission bands to be a more robust estimator of the quantity of a particular spectral form than its deconvolved intensity.

\section{Experimental}

Fluorescence probe

The fluorescence probe used for our experiments was the FE dye [14], a 3HC derivative 4'-diethylanino-3-hydroxyflavone. The class of $3 \mathrm{HC}$ derivatives was selected due to (1) the dramatic wavelength-ratiometric response of their fluorescence spectra to both polarity and H-bonding [15] of the microenvironment; (2) their increasingly popular application as fluorescence sensors and probes; and (3) the fact that they conform ideally to the model of the ground-state and excited-state behavior under consideration. In the presence of H-bond proton donors (e.g. hydroxyl groups) such probes exhibit two ground state forms, $\mathrm{N}$ (normal) and $\mathrm{H}-\mathrm{N}$, in which the 4-carbonyl serving as acceptor is H-bonded to the proton donor [16]. Upon selective excitation of the $\mathrm{N}$ form to the state $\mathrm{N}^{*}$, a picosecondrange Excited State Intramolecular Proton Transfer (ESIPT) reaction generates the tautomeric $\mathrm{T}^{*}$ form with a strongly red-shifted fluorescence band. The $\mathrm{T}^{*}$ band is predominantly observed in low-polar media whereas the $\mathrm{N}^{*}$ band dominates in media of high polarity, so that the $\mathrm{N}^{*} / \mathrm{T}^{*}$ intensity ratio $\left(I_{\mathrm{N}^{*}} / I_{\mathrm{T}^{*}}\right)$ taken at the band maxima is a strong indicator of polarity [14]. In contrast, the $\mathrm{H}-\mathrm{N}^{*}$ form does not exhibit rapid tautomerization on the timescale of emission, and its presence in emission characterizes the H-bonding proton-donor ability of the environment, particularly hydration in biological systems (Fig. 4).

The intensity ratio $I_{\mathrm{N}^{*}} / I_{\mathrm{T}^{*}}$ is known to correlate strongly with the polarity of the medium [17]. Indeed, there exists a

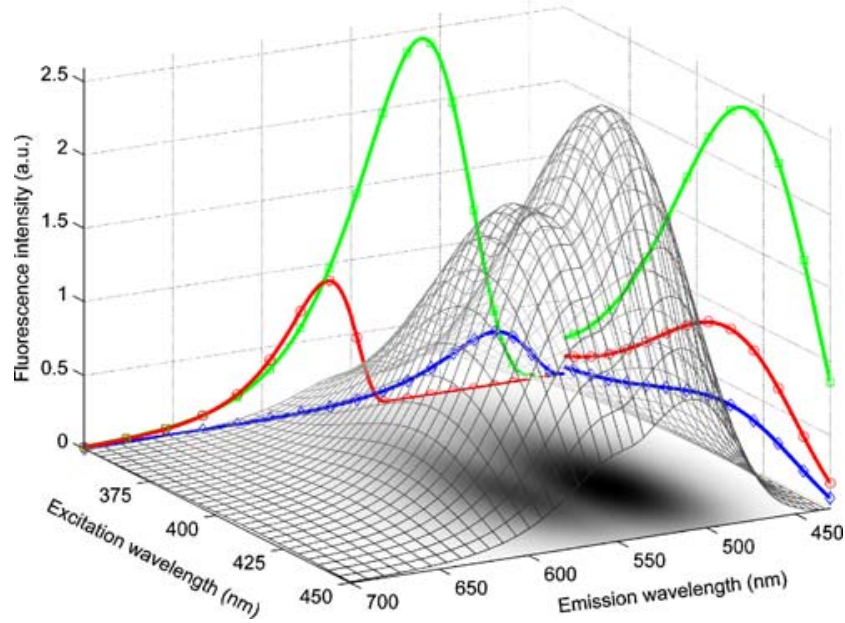

Fig. 3 Three-band deconvolved 2D fluorescence spectrum in the case of strong overlap between individual bands. The combined spectrum is represented as a 2D surface and mesh, while the individual emission and excitation bands (at their respective excitation and emission maxima) are projected onto the sides. The data are for FE dye in octanol, with $\mathrm{N}^{*}$ (blue diamonds), $\mathrm{H}-\mathrm{N}^{*}$ (green squares) and $\mathrm{T}^{*}$ (red circles) bands 


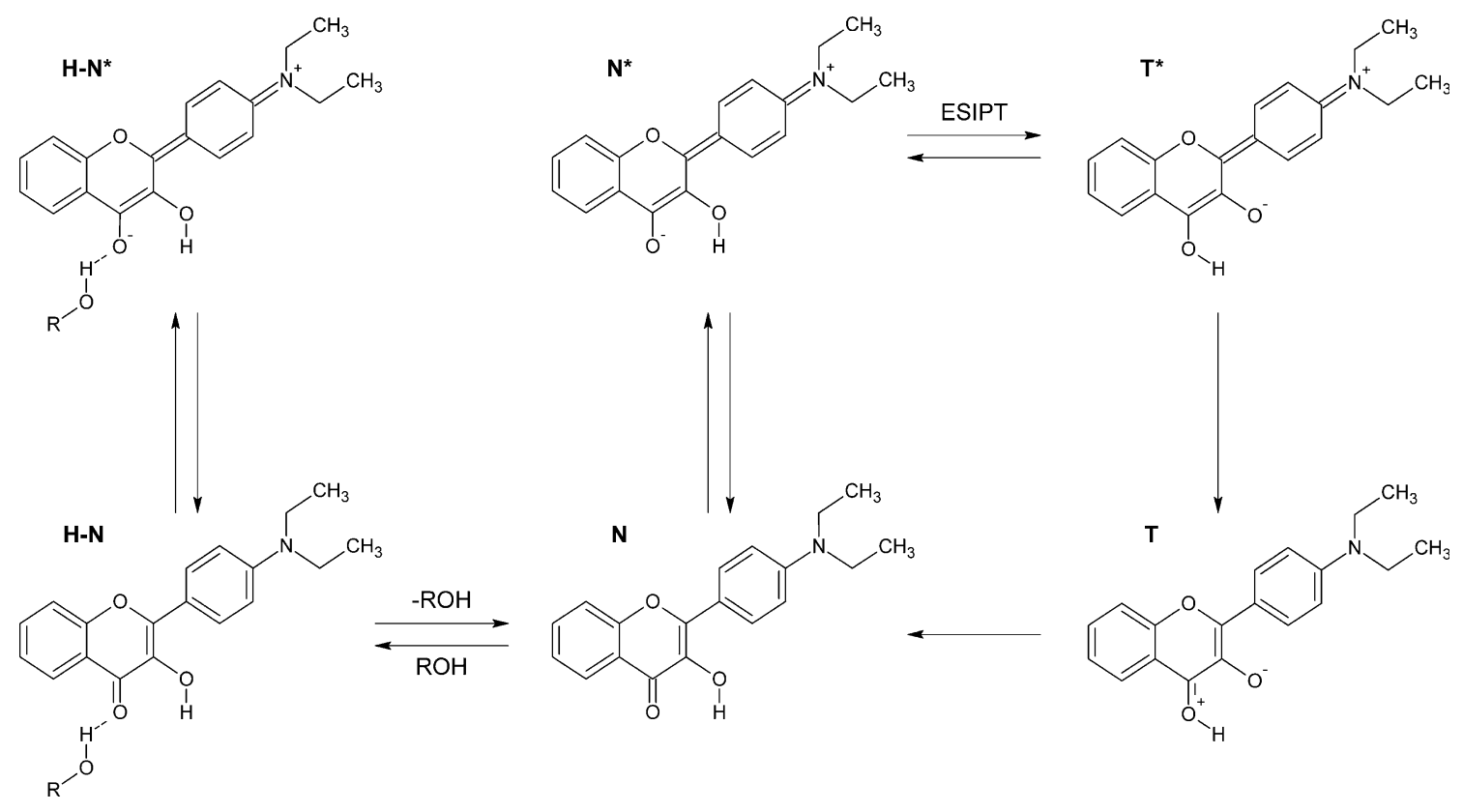

Fig. 4 Ground-state and excited-state transformations of the FE dye

linear dependence of $\log _{10}\left(I_{N^{*}} / I_{T^{*}}\right)$ on the solvent polarity function $f(\varepsilon)=(\varepsilon-1) /(2 \varepsilon+1)$, where $\varepsilon$ is the dielectric constant. Such a dependence is in accordance with a continuous model of dielectric relaxation that describes the physical basis of polarity [18]. This ratiometric fluorescence response has proven to be very useful for estimating relative polarities and dielectric constants in lipid vesicles $[19,20]$ and hydrophobic pockets in bovine serum albumin [21].

Hydrogen bonding results in the appearance of an additional ground-state $\mathrm{H}-\mathrm{N}$ form that can be excited and thus generates a third emitting species. Since this form exists in dynamic equilibrium with the H-bond free form, its presence is a good quantitative measure for the H-bonding potential of the environment [16]. The establishment of the $\mathrm{N}^{*} \leftrightarrow \mathrm{T}^{*}$ equilibrium occurs rapidly on the time scale of emission (tens of picoseconds) [16], whereas the distribution between $\mathrm{H}$-bonded and $\mathrm{H}$-bond free forms is retained in the excited state, thereby determining the relative intensities of the corresponding emission bands.

As a consequence of these coupled phenomena, we observe two forms $(\mathrm{N}$ and $\mathrm{H}-\mathrm{N})$ in excitation and three forms $\left(\mathrm{N}^{*}, \mathrm{H}-\mathrm{N}^{*}\right.$ and $\left.\mathrm{T}^{*}\right)$ in the emission spectra. The overlapping locations hinder their selective detection. The $\mathrm{H}-\mathrm{N}^{*}$ excitation band exhibits only a small shift to longer wavelengths relative to the $\mathrm{N}^{*}$ band. Accordingly, its fluorescence band is also shifted, occupying a position between the $\mathrm{N}^{*}$ and $\mathrm{T}^{*}$ fluorescence bands. Our task is to resolve by deconvolution the experimentally recorded fluorescence spectra into the three underlying components.
Experiments in model solvents

The FE probe was kindly provided by Dr. AS Klymchenko (Louis Pasteur University, Strasbourg, France). The dye was synthesized, purified and characterized as indicated previously [14]. The tabulated spectra from this publication were subjected to analysis. In addition, focused experiments were performed in several solvents and solvent mixtures. The investigated solvents were dimethyl sulfoxide (DMSO), acetone, octanol, and chloroform. Mixtures of ethyl acetate (EtAc) and water at $0 \%, 1 \%, 2 \%$ and $3 \%$ water additions were measured as well. Emission spectra were acquired with a Cary Eclipse spectrofluorimeter (Varian, Australia) equipped with a thermally controlled cuvette holder. They were corrected for wavelengthdependent excitation intensity and detection efficiency.

\section{Results and discussion}

The deconvolution method was tested in the analysis of the spectra of a 3-hydroxychromone (3HC) derivative in model solvents and solvent mixtures. In the case of only one ground-state form present in the system, the emission spectrum, even if represented by two bands, should not depend on the excitation wavelength. The validity of this statement was confirmed in control experiments of FE dye dissolved in neat ethyl acetate. As expected, when deconvolved into two completely separate bands (14 parameters, $\left.R^{2}=0.995\right)$, the shapes of the two fitted 
Table 1 Fitting parameters for the $\mathrm{N}^{*}$ and $\mathrm{T}^{*}$ excitation bands in neat ethyl acetate, showing only minimal deviation

\begin{tabular}{lcccc}
\hline Band & $\lambda_{0, \text { ex }}(\mathrm{nm})$ & $H_{\text {ex }}\left(\mathrm{cm}^{-1}\right)$ & $\rho_{\mathrm{ex}}$ & $I_{0}$ \\
\hline $\mathrm{N}^{*}$ & 402 & $3,720 \pm 80$ & $1.47 \pm 0.07$ & $0.49 \pm 0.01$ \\
$\mathrm{~T}^{*}$ & 401 & $3,640 \pm 20$ & $1.51 \pm 0.02$ & $2.53 \pm 0.02$ \\
Combined N* $^{*}$ and T* & 401 & $3,650 \pm 20$ & $1.50 \pm 0.02$ & $0.50 \pm 0.01$ \\
& & & & $2.53 \pm 0.02$ \\
\hline
\end{tabular}

Error margins are $95 \%$ confidence intervals of the fit. When not given, they are smaller than the implied precision

excitation bands deviated only minimally, as shown in Table 1. As a comparison, the parameters for a fit with just a single excitation band (11 parameters, $\left.R^{2}=0.995\right)$ is given as well.

\section{H-bond proton donor sensitivity of dye response}

When deconvolving EtAc-water mixtures, we constrained the shapes and positions of the bands to be the same across all mixtures, with only the relative intensities changing between the different experiments. We therefore fit 44 complete emission spectra with only 27 parameters $(18+3$ extra intensities per mixture), obtaining a robust result without specifying any additional constraints $\left(R^{2}=0.996\right)$. Table 2 summarizes the results of this fit. Since the addition of small quantities of water causes formation of H-bonds without significant change of polarity, the persistence of the $I_{\mathrm{N}^{*}} / I_{\mathrm{T}^{*}}$ ratio should be a test of the goodness of the fit. This expectation was fulfilled in that the $I_{\mathrm{N}^{*}} / I_{\mathrm{T}}$ ratio showed only small changes $(0.207,0.266,0.227,0.175$ for the 0 $3 \%$ water concentrations), whereas the $\mathrm{H}-\mathrm{N} *$ band intensity changed dramatically (from 0 to 2.11).

The emission spectra and their deconvolution at $400 \mathrm{~nm}$ excitation are given in Fig. 5 while Fig. 6 shows the virtual excitation spectra of the resolved bands, constructed by plotting the peak emission intensities of the three bands for all excitation wavelengths. The red-shifted (by $\sim 5 \mathrm{~nm}$ ) band corresponding to excitation of the $\mathrm{H}-\mathrm{N}$ form can be distinguished clearly only by varying the emission wavelength. Its contribution increases dramatically as a function of the water concentration, whereas the $\mathrm{N}^{*} / \mathrm{T}^{*}$ intensity ratio remains relatively constant.

Polarity probing in the presence of $\mathrm{H}$-bond proton donors

We also deconvolved the fluorescence spectra of FE in four neat solvents (DMSO, acetone, octanol, chloroform), in order to relate our results to previous work with this compound [14]. The dye in DMSO and acetone, being aprotic solvents that do not possess hydrogen-bond donating groups, did not exhibit a red-shifted excitation band (fitted shift less than $2 \mathrm{~nm}$ ). As expected, the emission in this case could be fitted with two bands. In contrast, the excitation spectra in octanol and chloroform displayed a two-band behavior and required three-band fitting in emission. For these fits we applied additional constraints: $2,500 \leq H_{e m} \leq 3,500$ ( $\mathrm{N}^{*}$ and $\mathrm{H}-\mathrm{N}^{*}$ bands) and the three band positions constrained to within $50 \mathrm{~nm}$ of their expected positions. Table 3 summarizes the results. The fits were stable, except for some uncertainty in the $\mathrm{N}^{*}$ band for the three-band fits of octanol and chloroform, as it appears only as a shoulder on the larger $\mathrm{H}-\mathrm{N}^{*}$ band.

In a previous publication [14], in which the fluorescence spectra were analyzed with only two bands, the $I_{\mathrm{N} *} / I_{\mathrm{T} *}$ ratio for octanol and chloroform did not fit the same solvent polarity function $f(\varepsilon)=(\varepsilon-1) /(2 \varepsilon+1)$ constructed from the data for other (neutral or hydrogen bond acceptor) solvents. By deconvolving those spectra with three bands, thereby separating the influence of the hydrogen bond donor ability of the solvent, the data fall on the same line as

Table 2 Parameters for the fitting of FE dye excitation and emission spectra in EtAc-water mixtures

\begin{tabular}{|c|c|c|c|c|c|c|c|c|c|c|}
\hline \multirow[t]{2}{*}{ Band } & \multirow{2}{*}{$\begin{array}{l}\lambda_{0, \mathrm{em}} \\
(\mathrm{nm})\end{array}$} & \multirow[t]{2}{*}{$H_{\mathrm{em}}\left(\mathrm{cm}^{-1}\right)$} & \multirow[t]{2}{*}{$\rho_{\mathrm{em}}$} & \multirow[t]{2}{*}{$\lambda_{0, \mathrm{ex}}(\mathrm{nm})$} & \multirow[t]{2}{*}{$H_{\mathrm{ex}}\left(\mathrm{cm}^{-1}\right)$} & \multirow[t]{2}{*}{$\rho_{\mathrm{ex}}$} & \multicolumn{4}{|l|}{$I_{0}$} \\
\hline & & & & & & & $0 \%$ & $1 \%$ & $2 \%$ & $3 \%$ \\
\hline $\mathrm{N}^{*}$ & $483 \pm 2$ & $3,080 \pm 70$ & $0.79 \pm 0.03$ & 402 & 3,650 & 1.49 & $0.54 \pm 0.03$ & $0.56 \pm 0.03$ & $0.48 \pm 0.04$ & $0.40 \pm 0.07$ \\
\hline $\mathrm{T}^{*}$ & 572 & 1,570 & 0.62 & “ & “ & “ & $2.61 \pm 0.01$ & $2.10 \pm 0.01$ & $2.11 \pm 0.01$ & $2.29 \pm 0.02$ \\
\hline $\mathrm{H}-\mathrm{N}^{*}$ & 503 & $2,930 \pm 30$ & 0.74 & 407 & $3,610 \pm 20$ & $1.47 \pm 0.02$ & $0.00 \pm 0.04$ & $0.43 \pm 0.04$ & $1.16 \pm 0.04$ & $2.11 \pm 0.06$ \\
\hline
\end{tabular}

The parameters for the $\mathrm{N}^{*}$ and $\mathrm{T}^{*}$ excitation bands are the same. Error margins are the $95 \%$ confidence intervals of the fit. When not given, they are smaller than the implied precision 

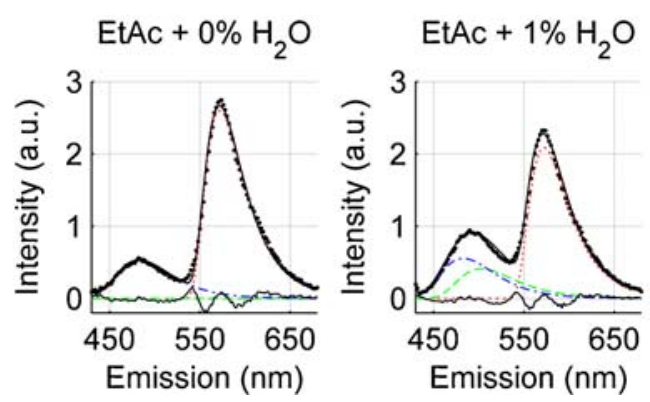

Fig. 5 EtAc-water mixtures: FE dye emission spectra and their deconvolution at $400 \mathrm{~nm}$ excitation. The broken colored lines are the deconvolved bands (blue dash-dot for $\mathrm{N}^{*}$, green dash for $\mathrm{H}-\mathrm{N}^{*}$, and

the other solvents (Fig. 7). As discussed above, we consider the band areas $\left(A_{\mathrm{N}^{*}}\right.$ and $\left.A_{\mathrm{T}^{*}}\right)$ to be more informative than simple intensities. Thus, we recalculated the original plot by reprocessing the original spectra, but using the obtained areas, calibrated so as to achieve comparable fluorescence intensities across the different experiments. An important consequence follows from this analysis. When the contribution of the $\mathrm{H}-\mathrm{N}^{*}$ form is taken into account, the $A_{\mathrm{N}^{*}} / A_{\mathrm{T}^{*}}$ ratio constitutes a direct indicator of polarity in both aprotic and $\mathrm{H}$-bonding environments, whereas the relative fraction of the $\mathrm{H}-\mathrm{N}^{*}$ form is an indicator of such $\mathrm{H}$-bonding.

On a broader-scale application of the algorithm

Organic dyes exhibiting both ground-state and excited-state transformations are presently the most efficient probes for extracting two or more uncoupled molecular parameters by spectroscopy. The challenge is to devise "smart fluorophores" able to realize this concept based on different mechanisms. In the ground state, not only H-bonding but also isomerizations, tautomerizations, protonation/deprotonation and charge transfers can generate a two-state response of fluorescence dyes. Corresponding excited state reactions include intramolecular charge transfer (ICT), photoisomerizations as well as intramolecular or intermo-
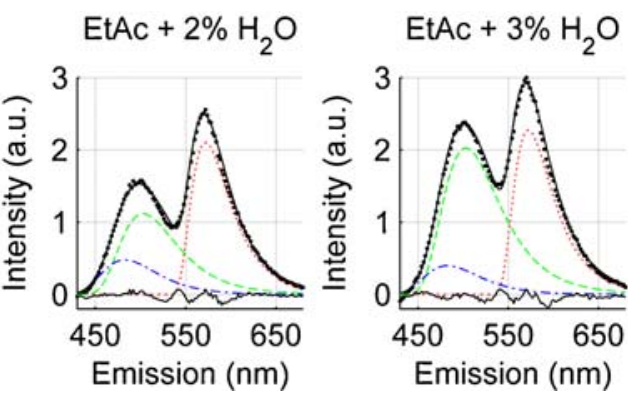

red dots for $\mathrm{T}^{*}$ ) and the upper solid black line is the sum of $\mathrm{N}^{*}$, $\mathrm{H}-\mathrm{N}^{*}$, and $\mathrm{T}^{*}$. The dotted black line represents the experimental data, while the lower solid black line is the residual

lecular proton transfer [22]. The following (a, b c) are examples of such reactions.

(a) Dyes existing as ground-state conformers, so that only one of the conformers exhibits an excited-state reaction. Commonly, only the conformer with a planar conformation of molecular segments undergoes excited-state ESIPT, resulting in the observation of both initially excited and tautomer emissions. A variety of such reactions exist and a suitable tool for their analysis is needed. Whereas the ESIPT equilibrium and isomerism are strongly sensitive to polarity [23] and intermolecular H-bonding, the ground-state conformation equilibrium can shift upon adsorption to macromolecular structures or interfaces.

In dyes with conformational flexibility, only the conformer with an intact internal H-bond between donor and acceptor groups can exhibit ESIPT. In protic media this bond is often disrupted due to stabilization by H-bonding with the solvent $[24,25]$. Thus, the shifts in ground-state equilibrium can report on $\mathrm{H}$-bonding potential.

(b) Dyes exhibiting protic equilibrium in the ground state, so that only one of the $\mathrm{pH}$-dependent forms can participate in the excited-state reaction. Examples of such behavior is exhibited by $3 \mathrm{HC}$ derivatives with
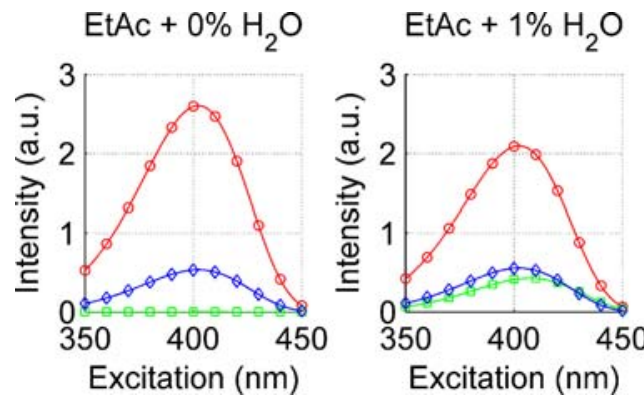

Fig. 6 EtAc-water mixtures: virtual excitation spectra of FE dye, obtained by deconvolution. Note the red-shifted position of the $\mathrm{H}-\mathrm{N}^{*}$ band (green squares), and the increasing $\mathrm{H}-\mathrm{N}^{*}$ band contribution

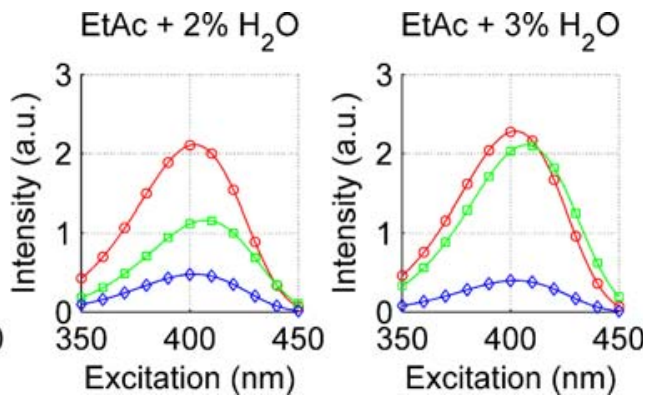

while the intensity ratio between emissions collected at $\mathrm{N}^{*}$ (blue diamonds) and $\mathrm{T}^{*}$ (red circles) bands remains roughly equal 
Table 3 Fitting parameters for FE dye in neat solvents. Error margins are the $95 \%$ confidence intervals of the fit. When not given, they are smaller than the implied precision

\begin{tabular}{|c|c|c|c|c|c|c|c|c|}
\hline Solvent & Band & $\lambda_{0, \mathrm{em}}(\mathrm{nm})$ & $H_{\mathrm{em}}\left(\mathrm{cm}^{-1}\right)$ & $\rho_{\mathrm{em}}$ & $\lambda_{0, \mathrm{ex}}(\mathrm{nm})$ & $H_{\mathrm{ex}}\left(\mathrm{cm}^{-1}\right)$ & $\rho_{\mathrm{ex}}$ & $I_{0}$ \\
\hline \multirow[t]{2}{*}{ DMSO } & $\mathrm{N}^{*}$ & 517 & $2,920 \pm 20$ & 0.89 & 410 & 3,770 & $1.42 \pm 0.01$ & $2.40 \pm 0.01$ \\
\hline & $\mathrm{T}^{*}$ & 592 & $1,620 \pm 20$ & 0.65 & “ & “ & “ & $1.23 \pm 0.02$ \\
\hline \multirow[t]{2}{*}{ Acetone } & $\mathrm{N}^{*}$ & 504 & $3,070 \pm 40$ & $0.89 \pm 0.02$ & 403 & $3,750 \pm 20$ & 1.40 & $0.95 \pm 0.01$ \\
\hline & $\mathrm{T}^{*}$ & 579 & 1,610 & $0.63 \pm 0.01$ & “ & “ & “ & $1.24 \pm 0.01$ \\
\hline \multirow[t]{3}{*}{ Octanol } & $\mathrm{N}^{*}$ & $471 \pm 10$ & $2,500 \pm 140$ & $0.67 \pm 0.2$ & 412 & $3,680 \pm 40$ & 1.48 & $0.36 \pm 0.06$ \\
\hline & $\mathrm{T}^{*}$ & 563 & $1,460 \pm 30$ & $0.62 \pm 0.01$ & “ & “ & “ & $0.88 \pm 0.02$ \\
\hline & $\mathrm{H}-\mathrm{N}^{*}$ & $511 \pm 1$ & $2,720 \pm 40$ & $0.78 \pm 0.02$ & 419 & $3,530 \pm 30$ & 1.52 & $2.44 \pm 0.2$ \\
\hline \multirow[t]{3}{*}{ Chloroform } & $\mathrm{N}^{*}$ & $471 \pm 20$ & $2,500 \pm 900$ & $0.82 \pm 0.1$ & 413 & $3,580 \pm 30$ & $1.55 \pm 0.03$ & $0.27 \pm 0.1$ \\
\hline & $\mathrm{T}^{*}$ & 564 & $1,500 \pm 30$ & $0.62 \pm 0.01$ & “ & “ & “ & $1.27 \pm 0.02$ \\
\hline & $\mathrm{H}-\mathrm{N}^{*}$ & $490 \pm 4$ & $2,760 \pm 160$ & $0.57 \pm 0.1$ & $416 \pm 1$ & $3,650 \pm 70$ & $1.62 \pm 0.07$ & $0.52 \pm 0.2$ \\
\hline
\end{tabular}

intense two-band emission in water [26]. As in the case studied in the present report, the fluorescence band that is strongly shifted to longer wavelengths originates from an ESIPT reaction. An increase of $\mathrm{pH}$ from 7 to 9 leads to a new spectral form, which becomes dominant. It exhibits an absorption and fluorescence spectrum strongly shifted to the red and an emission intensity decreased by $>50 \%$. The origin of this transformation is the dissociation of a proton from the 3-OH group, such that ESIPT is no longer possible in the excited state. Whereas the ratio of intensities of the $\mathrm{N}^{*}$ and $\mathrm{T}^{*}$ forms remains an indicator of polarity, the relative magnitude of the new form can serve as a $\mathrm{pH}$ indicator.

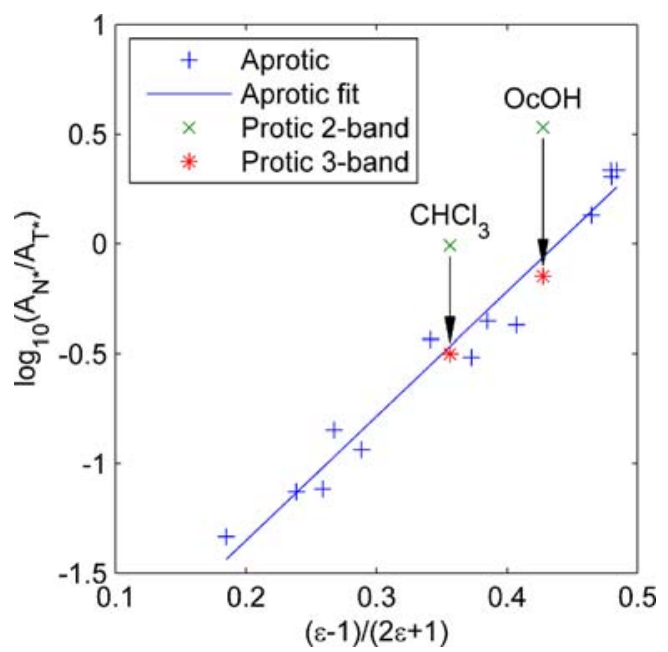

Fig. $7 \log _{10}$ of the ratio of the areas of $\mathrm{N}^{*}$ and $\mathrm{T}^{*}$ bands $\left(A_{\mathrm{N}^{*}} / A_{\mathrm{T}^{*}}\right)$ as a function of solvent polarity function $f(\varepsilon)$, showing a linear relationship. The solvents are (from left to right): hexane, toluene, carbon disulfide, thiophene, d-n-butyl ether, anisole, chloroform, bromobenzene, ethyl acetate, tetrahydrofuran, octanol, acetone, N,N-dimethylformamide, acetonitrile, and dimethyl sulfoxide (data from [14] kindly provided by Dr. AS Klymchenko). The values for octanol $(\mathrm{OcOH})$ and chloroform $\left(\mathrm{CHCl}_{3}\right)$ are given for both the original 2-band and improved 3-band deconvolution, the shift in results being indicated by arrows
The 3-OH dissociated (anionic) form of 3HC derivatives can be observed not merely in water at alkaline $\mathrm{pH}$. It is also present at neutral $\mathrm{pH}$ upon binding of 3-hydroxyflavone to serum albumin [27] and upon incorporation of a close analog of $\mathrm{FE}$, probe $\mathrm{F}$, into phosphatidylglycerol vesicles in HEPES buffer [28]. In these cases, the proton transfers not to the solvent but to a closely located strong proton acceptor. Thus, together with polarity, the fluorescence response can provide information on the proton-acceptor ability of the binding sites.

(c) Dyes exhibiting different protic equilibria in the excited and ground states. Many organic molecules that are able to dissociate protons do so with greater ease in the excited state due to a dramatic decrease of their $\mathrm{pK}$ values. Within the range of $\mathrm{pH}$ values such 'photoacids' possess two bands in their emission spectra corresponding to a common excitation spectrum. Such an ability to dissociate can be modulated by ground-state interactions and suppressed in media of low polarity [29]. Therefore, dyes with significant ground-state charge transfer and photoacidic groups (e.g. aromatic hydroxyl groups) can serve as both polarity and $\mathrm{pH}$ indicators.

Excited-state proton removal from a photoacidic group can occur even in hydrophobic media if a strong proton acceptor (e.g. primary amine) is present. Solutions of $3 \mathrm{HC}$ dyes in toluene in the presence of an amine show three emission bands corresponding to the $\mathrm{N}^{*}, \mathrm{~T}^{*}$ and anionic forms [30]. The information content of such a response can be increased by using dyes containing two ESIPT sites [31].

In summary, a combination of ground-state and excitedstate effects implemented into smart fluorescence probes can provide rich, multiparametric information. Obtaining this information requires global deconvolution involving both the excitation and emission spectra. We have applied the formalisms developed in this report to the analysis of complexes of ESIPT dyes with the protein $\alpha$-synuclein in amyloid fibrils [32]. 


\section{Conclusions}

In general, the deconvolution of a complex fluorescence spectrum into discrete components is carried out in the absence of an attempt (or formal instrument) for introducing additional physically motivated restrictions to reduce ambiguity. Such an approach is successful only if the spectral positions and shapes of the components are well known or do not change with variation of the properties of the system. In the absence of this information, the spectroscopic parameters are often fixed to values obtained by physical intuition or obtained under conditions different than those corresponding to the analyzed spectrum. The data acquired in simplified systems, such as neat solvents and their mixtures, are often used in studies of micro-heterogeneous and biological systems. A different approach is demonstrated in this work. In order to avoid arbitrary and uncertain fixing of input parameters and to obtain more stable and reliable results we implemented a 2D excitation-emission global deconvolution analysis and applied constraints based on the photophysical mechanism of the excited-state process. Appropriate deconvolution of the fluorescence spectrum into three individual components served to resolve the spectral location and contribution of the emission bands of a 3-hydroxychromone probe, which could be interpreted in terms of polarity and H-bonding potential of the environment. In this manner, the population distribution between its different molecular states and locations in heterogeneous systems was assessed.

Acknowledgements We thank Dr. A.S. Klymchenko for providing the FE dye, the spectral data from [14], and useful discussions. MSC was awarded a postdoctoral fellowship from the Alexander von Humboldt Foundation. This work was supported by the DFG Center for Molecular Physiology of the Brain (DFG CMPB), Cluster of Excellence 171 of the CMPB, in Göttingen, Germany, and the Max Planck Society (Toxic Protein Conformation project).

Open Access This article is distributed under the terms of the Creative Commons Attribution Noncommercial License which permits any noncommercial use, distribution, and reproduction in any medium, provided the original author(s) and source are credited.

\section{References}

1. Demchenko AP (2009) Introduction to fluorescence sensing. Springer, Netherlands

2. Klymchenko AS, Demchenko AP (2008) Multiparametric probing of microenvironment with solvatochromic fluorescent dyes. Methods Enzymol 450:37-58. doi:10.1016/S0076-6879(08)03403-4

3. Tichonov AN, Arsenin VY (1974) Methods of solving incorrect problems. Nauka, Moscow

4. Birks JB (1970) Photophysics of aromatic molecules. Wiley Interscience, New York

5. Nemkovich N, Rubinov A, Tomin V (1991) In: Lakowicz JR (ed) Topics in fluorescence spectroscopy. Springer, USA, pp 367-428
6. Kalauzi A, Mutavdzic D, Djikanovic D, Radotic K, Jeremic M (2007) Application of asymmetric model in analysis of fluorescence spectra of biologically important molecules. J Fluoresc 17 (3):319-329. doi:10.1007/s10895-007-0175-3

7. Barker BE, Fox MF (1980) Computer resolution of overlapping electronic absorption-bands. Chem Soc Rev 9(2):143-184. doi:10.1039/cs9800900143

8. Siano DB, Metzler DE (1969) Band shapes of electronic spectra of complex molecules. J Chem Phys 51(5):1856. doi:10.1063/ 1.1672270

9. Burstein EA, Abornev SM, Reshetnyak YK (2001) Decomposition of protein tryptophan fluorescence spectra into log-normal components. I. Decomposition algorithms. Biophys J 81(3):16991709. doi:10.1016/S0006-3495(01)75823-7

10. Burstein EA, Emelyanenko VI (1996) Log-normal description of fluorescence spectra of organic fluorophores. Photochem Photobiol 64(2):316-320. doi:10.1111/j.1751-1097.1996.tb02464.x

11. Metzler R, Ambjornsson T (2005) Sensing DNA-DNA as nanosensor: a perspective towards nanobiotechnology. J Comput Theor Nanoscience 2(3):389-395. doi:10.1166/jctn.2005.209

12. Djikanovic D, Kalauzi A, Jeremic M, Micic M, Radotic K (2007) Deconvolution of fluorescence spectra: contribution to the structural analysis of complex molecules. Colloids Surf B Biointerfaces 54(2):188-192. doi:10.1016/j.colsurfb.2006.10.015

13. Demchenko AP (2002) The red-edge effects: 30 years of exploration. Luminescence 17(1):19-42. doi:10.1002/bio.671

14. Klymchenko AS, Demchenko AP (2003) Multiparametric probing of intermolecular interactions with fluorescent dye exhibiting excited state intramolecular proton transfer. Phys Chem Chem Phys 5(3):461-468. doi:10.1039/b210352d

15. Pivovarenko VG, Wroblewska A, Błazejowski J (2005) 2-[4(Dimethylamino)phenyl]-3-hydroxy-4H-chromene-4-one: A Hbond-sensitive fluorescent probe for investigating binary mixtures of organic solvents. Anal Chim Acta 545:74-78. doi:10.1016/j. aca.2005.04.042

16. Shynkar VV, Klymchenko AS, Piemont E, Demchenko AP, Mely Y (2004) Dynamics of intermolecular hydrogen bonds in the excited states of 4'-dialkylamino-3-hydroxyflavones. On the pathway to an ideal fluorescent hydrogen bonding sensor. J Phys Chem A 108(40):8151-8159. doi:10.1021/jp0479901

17. Ercelen S, Klymchenko AS, Demchenko AP (2002) Ultrasensitive fluorescent probe for the hydrophobic range of solvent polarities. Anal Chim Acta 464(2):273-287. doi:10.1016/S0003-2670(02) 00493-2

18. Suppan P, Ghoneim N (1997) Solvatochromism. Royal Society of Chemistry, Cambridge

19. Klymchenko AS, Duportail G, Demchenko AP, Mely Y (2004) Bimodal distribution and fluorescence response of environmentsensitive probes in lipid bilayers. Biophys J 86(5):2929-2941. doi:10.1016/S0006-3495(04)74344-1

20. Klymchenko AS, Mely Y, Demchenko AP, Duportail G (2004) Simultaneous probing of hydration and polarity of lipid bilayers with 3-hydroxyflavone fluorescent dyes. Biochim Biophys Acta 1665(1-2):6-19. doi:10.1016/j.bbamem.2004. 06.004

21. Ercelen S, Klymchenko AS, Demchenko AP (2003) Novel twocolor fluorescence probe with extreme specificity to bovine serum albumin. FEBS Lett 538(1-3):25-28. doi:10.1016/S0014-5793 (03)00116-9

22. Mallick A, Purkayastha P, Chattopadhyayay N (2007) Photoprocesses of excited molecules in confined liquid environments: An overview. J Photochem Photobiol C 8:109-127. doi:10.1016/j. jphotochemrev.2007.06.001

23. Brenlla A, Rodriguez-Prieto F, Mosquera M, Rios MA, Rios Rodriguez MC (2009) Solvent-modulated ground-state rotamer- 
ism and tautomerism and excited-state proton-transfer processes in o-hydroxynaphthylbenzimidazoles. J Phys Chem A 113(1):56-67. doi:10.1021/jp8076003

24. Das SK, Krishnamoorthy G, Dogra SK (2000) Excited state intramolecular proton transfer in 2-(2'-hydroxyphenyl)-1Hnaphth-[2, 3-d]-imidazole: effects of solvents and $\mathrm{pH}$. Can J Chemistry-Revue Canadienne De Chimie 78(2):191-205. doi:10.1139/cjc-78-2-191

25. Abou-Zied OK, Jimenez R, Thompson EHZ, Millar DP, Romesberg FE (2002) Solvent-dependent photoinduced tautomerization of 2-(2'-hydroxyphenyl)benzoxazole. J Phys Chem A 106(15):36653672. doi:10.1021/jp013915o

26. Klymchenko AS, Demchenko AP (2004) 3-Hydroxychromone dyes exhibiting excited-state intramolecular proton transfer in water with efficient two-band fluorescence. N J Chem 28(6):687692. doi: $10.1039 / \mathrm{b} 316149 \mathrm{~h}$

27. Sytnik A, Litvinyuk I (1996) Energy transfer to a proton-transfer fluorescence probe: tryptophan to a flavonol in human serum albumin. Proc Natl Acad Sci USA 93(23):12959-12963. doi:10.1073/pnas.93.23.12959

28. Shynkar VV, Klymchenko AS, Mely Y, Duportail G, Pivovarenko VG (2004) Anion formation of 4'-(dimethylamino)-3- hydroxyflavone in phosphatidylglycerol vesicles induced by HEPES buffer: A steady-state and time-resolved fluorescence investigation. J Phys Chem B 108:18750-18755. doi:10.1021/ jp0467189

29. Mironczyk A, Jankowski A, Chyla A, Ozyhar A, Dobryszycki P (2004) Investigation of excited-state proton transfer in 2-naphthol derivatives included in Langmuir-Blodgett films. J Phys Chem A 108(25):5308-5314. doi:10.1021/jp035919u

30. Demchenko AP, Klymchenko AS, Pivovarenko VG, Ercelen S, Duportail G, Mely Y (2003) Multiparametric color-changing fluorescence probes. J Fluoresc 13(4):291-295. doi:10.1023/ A:1025302525771

31. Roshal AD, Moroz VI, Pivovarenko VG, Wroblewska A, Blazejowski J (2003) Spectral and acid-base features of 3,7-dihydroxy-2,8diphenyl-4H,6H-pyrano[3,2-g]chromene-4,6-dione (diflavonol) -a potential probe for monitoring the properties of liquid phases. J Org Chem 68:5860-5869. doi:10.1021/jo034200f

32. Celej MS, Caarls W, Demchenko AP, Jovin TM (2009) A triple emission fluorescent probe reveals distinctive amyloid fibrillar polymorphism of wild-type $\alpha$-synuclein and its familial Parkinson's disease mutants. Biochemistry 48(31):7465-7472. doi:10.1023/ bi9003843 